\title{
Aerial Robotics: State-based Conflict Detection and Resolution (Detect and Avoid) in High Traffic Densities and Complexities
}

\author{
Jacco M. Hoekstra ${ }^{1}$ (D) - Joost Ellerbroek ${ }^{1}$ \\ Accepted: 21 May 2021 / Published online: 5 June 2021 \\ (C) The Author(s) 2021
}

\begin{abstract}
Purpose of Review A lot of research into decentralised, state-based conflict detection and resolution, or detect and avoid algorithms has been executed. This paper explains the essential properties of state-based conflict detection and reviews the work in the context of applications for not only manned but also unmanned aerial vehicles, where this might be applied relatively soon. Recent Findings Lately, based on several reviews of a variety of published algorithms, a selection has been implemented and simulated in extremely high traffic densities for comparison.

Summary The modified voltage potential has been surprisingly efficient, even compared with more complex algorithms or adaptations, as is apparent from looking at macroscopic metrics like domino effect, efficiency and safety. This indicates that to this date, it is so far the most suitable algorithm for the detect and avoid role for unmanned aerial vehicles in urban airspaces, or other areas where a high density is expected.
\end{abstract}

Keywords Detect and avoid · Conflict detection · Conflict resolution · Modified voltage potential $\cdot$ Geovectoring $\cdot$ Urban traffic management $\cdot \mathrm{UTM} \cdot \mathrm{U}$-space $\cdot$ Capacity management $\cdot \mathrm{UAV} \cdot$ Drones

\section{Introduction}

Advances in low-weight, low volume electrical power storage and the further miniaturisation of electronics have contributed to the development of unmanned aerial vehicles (UAVs) for other purposes than recreation. Some applications ranging from the delivery small cargo in urban environments or remote areas, surveillance applications up to even transporting persons in an urban environment have become technically and economically feasible (ref Malik, ref technical PAV paper). As a result, a very large increase in the number of vehicles is foreseen, Eurocontrol expects 70,000 drones (UAVs) operating in the European Airspace [1]. In urban environments, this will lead to unprecedented concentrations of air traffic. To ensure safe and reliable operations, a lot of attention has to be given to collision avoidance by assuring sufficient spatial separation between all vehicles

This article is part of the Topical Collection on Aerial Robotics

Jacco M. Hoekstra

J.M.Hoekstra@tudelft.nl

Joost Ellerbroek

J.Ellerbroek@tudelft.nl

1 TU Delft, Delft, Netherlands at any given time. This process is called separation assurance, which continues to be the task of air traffic control (ATC) in traditional aviation. The large number of vehicles expected in urban airspace, however, demands a change in the way separation assurance works. The required shift to autonomous operations brings the separation assurance task to the domain of robotics and of emerging behaviour of distributed systems.

\section{Airborne Separation Assurance: De-centralisation of ATC}

In manned aviation, the task of separation assurance has been centralised for most non-recreational air traffic since the invention of radar. As stated in ICAO Annex 11, per sector of airspace, air traffic control as a central operator has the task of maintaining a safe and orderly flow of traffic in the sector. The level of control is specified by the classification of the airspace. In class A airspace, often used for commercial air traffic, this means among others [2]:

- A clearance by ATC is required to enter the airspace and for tactical deviations from the flight plan (altitude, speeds, heading changes and direct to).

- Air traffic control is responsible for separation assurance. 
- Only flights under instrument flight rules (IFR) are allowed.

To manage the air traffic control in this airspace, a flight plan has to be filed before the flight starts. This allows the systems of the different air traffic controllers to share information by exchanging these flight data. Air traffic control needs to balance between safety, i.e. maintaining separation, and efficiency, i.e. allowing a near-optimal execution of the flight in terms of fuel and time. One of the limitations in this central optimisation is that normally the mass of an aircraft as well as the cost index (the ratio of cost between fuel and time) is not shared with air traffic control because of the commercially confidential nature of these data. These data are, however, required to optimise the route.

In 1993, the RTCA Task Force 3 [3] proposed to decentralise the task of separation assurance for the phases of the flight where a conflict between two aircrafts is mainly a local problem as both the problem as well as the consequences of the solution is local. That the global effect of conflict resolution is minimal can be illustrated by considering an en-route conflict resolution. Even when looking ahead only $5 \mathrm{~min}$ for the conflict detection, the required minimal deviations in altitude and heading have a negligible effect on the time of arrival at the next waypoint and at the final approach fix (due to the cosine of small angles being almost one):

$$
\Delta s=\frac{V \Delta t_{\text {lookahead }}}{\cos \tan ^{-1}\left(\frac{\Delta x}{V \Delta t_{\text {lookahead }}}\right)}
$$

Typically for a maximum change of $5 \mathrm{~nm}$ at $400 \mathrm{kts}$ ground speed (similarly when both numbers are scaled down for UAVs), this means an extra path length of $1.1 \%$ over 5 min of the route. Vertically, the effect is negligible due the small vertical speeds and the conservation of energy. Therefore, unless the magnitude of the ground speed itself is changed significantly, the system-wide effect of local conflict resolution manoeuvres is minimal: it does not need to impact the flow of the traffic when the aircraft need to be sequenced closer to their destination.

Airborne separation assurance, also sometimes referred to as free flight, decentralises the air traffic management (ATM) system and is enabled by sharing position and velocity information between airspace users via ADS-B, which allows moving the separation assurance task to the cockpit crew. Decentralising this task has several advantages and disadvantages. A disadvantage is, for example, the resulting traffic patterns will be more chaotic, making it harder to partially control the traffic which is not self-separating or taking over centrally in case of an airspace-wide failure. One of the advantages is that during the de-confliction the confidential information about mass, cost index and other flight preferences can be used as this change of the trajectory takes place in the on-board systems. Using well-chosen, common rules of the air with the exchanged position and velocities of the potentially conflicting traffic can be chosen without any further communication, coordination or negotiation cycles to avoid the conflict and collision. This robustness avoids time-outs, handshaking or and the risk of delays and endless loops.

A major benefit of decentralising the separation task is that way in which the number of aircraft affects the required actions by the separation assurance system. For a decentralised system, this increases linearly instead of quadratically as with a centralised system (see next section). Another benefit is that there is no requirement to maintain oversight, allowing a more optimal flight by free routing and a continuous cruise climb. This can result in traffic patterns that would have been impossible to monitor and control centrally.

Indeed, several studies have shown that the capacity of an airspace, the efficiency as well as the safety increase when changing decentralised ATM system compared to today's centralised ATM [4-8].

\section{D Trajectory-based Operations and State-based CD\&R}

In the next decade, improving the centralised ATM system was explored to achieve similar benefits $[9,10]$. This resulted in the concept of trajectory-based operations as a means to allow more free routing. Here, the flight plan and the clearances merge. Trajectory-based operations assume a predictability for a horizon long enough to avoid endless renegotiations. Conflicts between aircraft, however, are currently hard to predict beyond the horizon of $5 \min$ [11]. By using a 4D closed-loop, the predictability is enforced and thus improved. When this results in a flight to become suboptimal, a new trajectory can be requested.

While it can be expected that 4D-TBO will be useful to create a safe and efficient traffic flow, it might still need a tactical conflict detection and resolution, using state-based information as a robust complement to the longer, more flow- and capacity-oriented de-confliction by using the intended trajectory or flight plan.

\section{UAV and Urban Airspace Implications for Separation Assurance}

In combination with assigning reserved blocks of airspace (geo-cages or geo-fences), this trajectory-based approach is also proposed for urban/UAV airspace. But can this approach be maintained when the predicted growth in traffic numbers become a reality? 
The average probability for a pair of two vehicles in a given airspace having a loss of separation is called $p_{2}$. This probability is among others a function of the route structure of the airspace and several other characteristics of the traffic (see section Metropolis/geovectoring), but this probability is independent of the number of vehicles $N$. The global conflict probability determined by the possible number of combinations times this probability:

$p_{c}=\left(\begin{array}{c}N \\ 2\end{array}\right) p_{2}=\frac{N(N-1)}{2} p_{2}=\left(\frac{1}{2} N^{2}-\frac{1}{2} N\right) p_{2}$

As can be seen by the $N^{2}$ in this equation, the global conflict rate increases quadratically with the number of vehicles in an airspace. This global conflict probability is a measure for the conflict rate and thus presents the load on a centralised separation assurance system, such as current ATM, a future 4D-TBO-based ATM system or any foreseen centralised UAV air traffic control system. This has direct consequences for the safety, reliability and capacity of such system on top of the global node of failure it presents.

For decentralised deconfliction system, the local conflict rate should be observed which follows a very different relation even though $p_{2}$ does not change. The conflict probability for any vehicle system is simply the product of the probability that two vehicles meet multiplied with the number of other vehicles:

$p_{l}=(N-1) p_{2}$

As a result, independent of the actual implementation of the system, for very large numbers of vehicles the quadratic of the global conflict rate, means that a centrally organised system will no longer be feasible when the number of vehicles becomes as large as predicted (see Fig. 1). The situation then becomes similar to, for

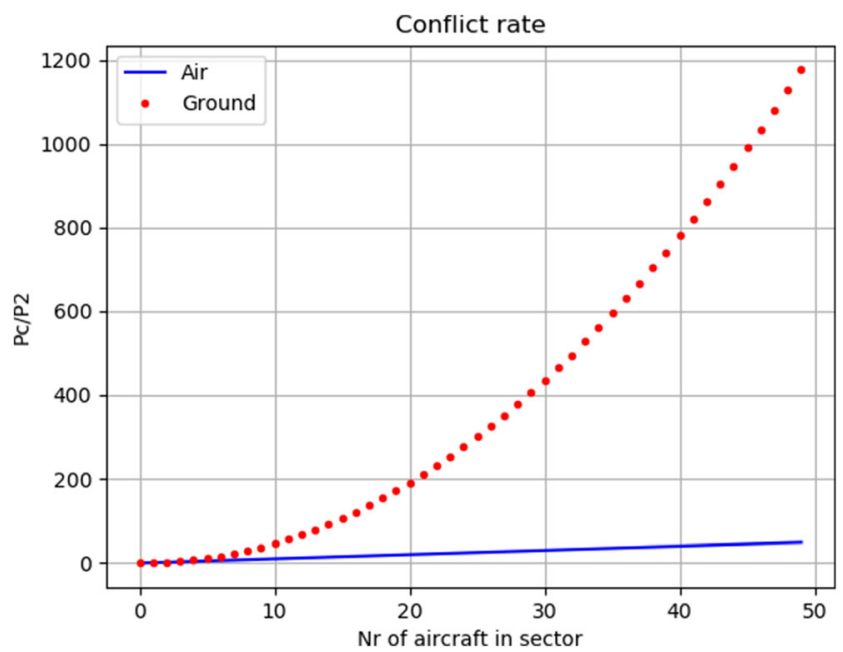

Fig. 1 Conflict rate shows difference in effect of traffic density for decentralised ("air") and centralise ("ground") separation assurance example, road traffic, which is also de-conflicted in a tactical and decentralised, or distributed, way.

The previous findings of the airborne separation assurance for conventional air traffic therefore have become very relevant for the detect and avoid of urban UAVs. The traffic volumes can only be handled with decentralisation, but how is currently still unknown.

This paper explores how what we do know about conflict detection, conflict resolution and conflict prevention can be employed in such a way that the UAVs will be operated safely and efficiently. The past and current studies aide in showing a possible direction forward to define separation assurance algorithms as well as capacity management for UAVs.

When discussing UAVs the conflict detection is often referred to as detect and avoid (D\&A). This is identical to conflict detection and resolution (CD\&R) and the same principles apply.

\section{Conflict Detection and Conflict Prevention}

When the positions of two aircrafts violate the separation requirement, it is a loss of separation (LoS). A conflict, however, is merely a predicted loss of separation within a given prediction horizon of lookahead time. To detect conflicts, the future paths of the aircraft need to be predicted. This can be done in a variety of ways, ranging from a linear extrapolation of the speed vector to using published flight plans or using transmitted intent information and/or autopilot settings.

State-based conflict detection uses estimated future trajectory, purely based on the position and extrapolating this with the current velocity vector, to get the trajectory in the immediate future.

Assume as a horizontal separation requirement a minimum distance $R$ is defined. In Fig. 2, two aircrafts are shown: the ownship, index 0 , with its position in the origin of our reference frame moving with a horizontal speed vector $\underline{v}_{0}$ and the potential intruder index, i, at position $\underline{x}_{i}$, moving with a speed vector $\underline{v}_{i}$.

The time left to the closest point of approach $t_{c p a}$ follows from Fig. 2:

$$
\begin{gathered}
\underline{v}_{r e l}=\underline{v}_{o}-\underline{v}_{i} ; \underline{x}_{r e l}=\underline{x}_{i} \\
\underline{v}_{r e l} \perp\left(\underline{x}_{r e l}-t_{c p a} \cdot \underline{v}_{r e l}\right) \Rightarrow \\
\underline{v}_{r e l} \cdot\left(\underline{x}_{r e l}-t_{c p a} \cdot \underline{v}_{r e l}\right)=0 \Rightarrow \\
t_{c p a}=\frac{\underline{v}_{r e l} \cdot \underline{x}_{r e l}}{\underline{v}_{r e l} \cdot \underline{v}_{r e l}}=\frac{\underline{v}_{r e l} \cdot \underline{x}_{r e l}}{\left|\underline{v}_{r e l}\right|^{2}}
\end{gathered}
$$

Once this time is known, using vector equations and/or the Pythagorean relation of the triangles, the minimum distance itself as well as the intrusion interval and intrusion entry and exit positions can easily be derived using the distance at CPA, 
Fig. 2 Calculating closest point of approach. The circle indicates the separation minimum as a protected zone

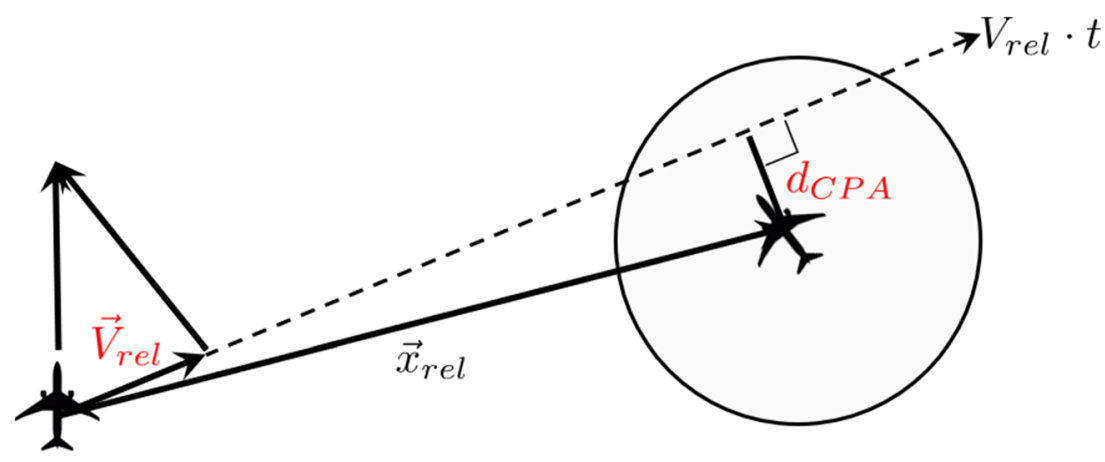

the length of the relative path through protected zone $\Delta \underline{x}_{i n t}$, and the required minimum separation, or radius of the protected zone R:

$$
\begin{gathered}
d_{c p a}=\left|\underline{x}_{i}-t_{c p a} \cdot \underline{v}_{r e l}\right| \\
\left|\Delta x_{\text {int }}\right|=2 \sqrt{R^{2}-d_{c p a}^{2}} \\
\Delta t_{\text {int }}=\frac{\left|\Delta x_{\text {int }}\right|}{\left|\underline{v}_{r e l}\right|}
\end{gathered}
$$

When the start of the conflict interval lies within the conflict prediction horizon or lookahead time, a conflict alert is triggered.

An effective way to investigate resolution possibilities to such a conflict is by observing the speed of the ownship relative to the intruding vehicle (see Fig. 3). To resolve the conflict, the speed vector of the ownship needs to change in such a way that the resulting relative speed falls outside of a conical area that has its origin at the position of the ownship, extending towards the intruding vehicle, touching the circle corresponding to the minimum separation distance.

The advantages of state-based conflict detection are its universal applicability, thanks to its unambiguous and straightforward definition, and its robustness against any deviations from a programmed trajectory or a cleared flight plan. Its flexibility and adaptability allow using it in flight operations for, e.g. surveillance operations, where the future trajectory could be unknown. The drawback is that it misses conflicts due to a change in the velocity vector.

A way to mitigate the false negatives in state-based conflict detection due to manoeuvring is to use a specific form conflict detection also as a means to prevent conflicts, by running the conflict detection logic on intended future speed vectors before turning or accelerating/decelerating to these new speeds by the aircraft that intends to manoeuvre. This could be defined as a new traffic rule:

- When not in conflict, the heading, vertical speed or speed vector shall not be changed in a way that it triggers a conflict within the lookahead time.

In manned aviation, this type of conflict prevention can be aided by adding non-intrusive bands on the heading, speed and vertical speed scales of the existing displays. This results in a simple visualisation of the 4D forbidden zones, but only in a one-dimensional way. This works well for changes of the flight state along only one of the variables heading or speed (or vertical speed) at the time, as is common practice.

\section{Conflict Resolution Algorithms}

The cone-shaped zone of "forbidden speeds" in Fig. 3 is only meaningful for the relative speeds which are different for each

Fig. 3 In the relative speed reference frame, the grey cone indicates the "forbidden" relative speeds that would lead to a loss of separation

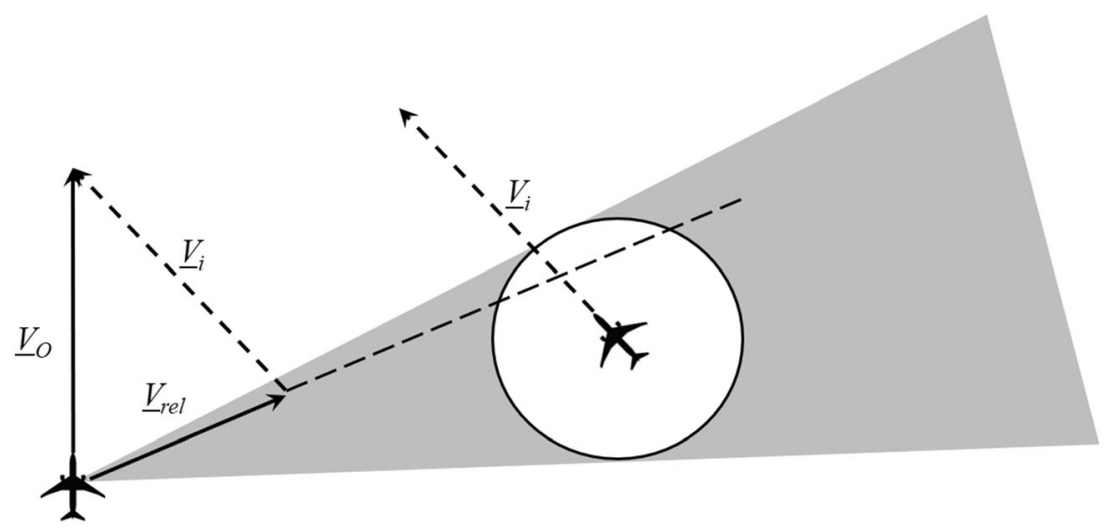


intruder as it includes the intruder's speed. To get to an absolute ownspeed reference frame, this cone is translated along the intruders speed vector in Fig. 4, so it indicates an area where we should not point with our own absolute speed vector.

$\underline{v}_{r e l}=\underline{v}_{0}-\underline{v}_{i} \quad($ relative speed reference frame)

$\underline{v}_{r e l}+\underline{v}_{i}=\underline{v}_{0} \quad$ (absolute speed reference frame)

In robotics, the resulting cone is known by the name velocity obstacle [12]. When for multiple intruders the velocity obstacle is known, we can select a speed vector, that is outside any velocity cone using very different algorithms, to steer free of all conflicts. A visualisation of these velocity obstacles is used in what is called the solution space diagram (SSD) in aviation-based VO research [13]. In this diagram, the open areas indicate potential solutions for all predicted conflicts. The SSD can also be seen as a two-dimensional extension of the one-dimensional bands of the Predictive ASAS system, allowing the identification of conflict resolution manoeuvres which require simultaneously changing speed and heading.

\section{Review Studies of Resolution Algorithms for $D \& A / C D \& R$}

A first overview of conflict detection and resolution algorithms was made in 2000 by Kuchar and Yang [14]. In 2016, a taxonomy of different conflict detection and resolution algorithms was published by Jenie et al. [15]. The most complete and recent overview was made in 2020 for both manned as well as unmanned aviation an up to date review by Ribeiro et al. [16••]. This last study has produced two tables which classify resolution algorithms for manned and unmanned aviation, even though many of the first category can be used for UAV operations as well.

\section{Variations of State-based Conflict Resolution}

To investigate these global effects of resolution methods, several metrics have been defined in literature, which are used to judge the overall result of CD\&R in three different categories:

- Safety: number of losses of separation and intrusion severity $[17 \cdot \bullet]$

- Efficiency: extra route flown, route efficiency or work done $[17 \cdot \bullet]$

- Stability: domino effect parameter (DEP) [18]

Ribeiro et al. defines three categories for decentralised methods [16••]: methods can resolve multi-aircraft conflicts sequentially (pairwise-sequential), they can search for a joint solution to all conflicts (joint solution), or they can take a (weighted) summation of the individual resolution vectors of each pairwise conflict (pairwise summed). An example of a method of the third category is the modified voltage potential algorithm.

\section{Modified Voltage Potential Algorithm}

The state-based modified voltage potential (MVP) algorithm studies have repeatedly shown very promising results in many of our studies, even in extreme traffic densities. The underlying principle of this method is based on an article by Eby from

Fig. 4 Velocity obstacle indicates the forbidden zone in the absolute ownspeed reference frame

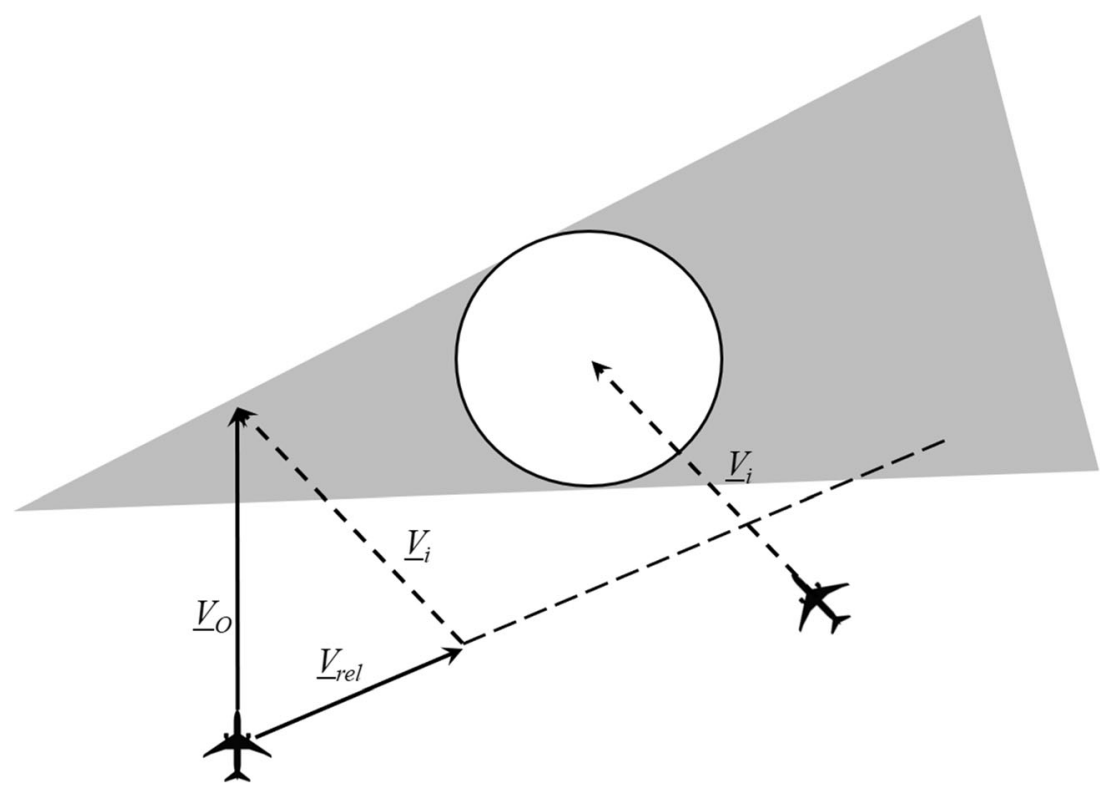


Lincoln Labs [19], which was adapted to make it suitable for airborne separation assurance by NLR and NASA [20].

Two features distinguish MVP from most other state-based algorithms:

- Implicit coordination based on geometry of conflict to always have a change of the speed vector, i.e. an avoidance vector, that increases the minimum distance at the closest point of approach.

- In case of multi-aircraft conflicts, the avoidance vectors (proposed changes of the speed vector) of the different conflicts are summed in a vector fashion, instead of looking for one-sided conflict-free speeds.

\section{Implicit Coordination by Geometry}

When two vehicles detect that they are in conflict with each other, and both start manoeuvring to solve it, the net effect can be adverse if both manoeuvres are not coordinated. It can even happen that a conflict is prolonged until a loss of separation can no longer be avoided. Such situations can be avoided by coordinating the manoeuvres of the involved actors. This is called explicit coordination when an additional exchange of messages is required. Explicit coordination has several drawbacks:

- Delay of the start of a conflict resolution manoeuvre, increases risk of loss of separation in very short-term conflicts

- Risk of creating many dependencies in case of multiaircraft conflicts, potentially even loops or deadlocks in severely congested cases

- Requires compatible systems also with respect to communicating intent

- Requires more bandwidth as for each conflict one or more coordination messages are required

A way to avoid the drawbacks of explicit coordination is to adopt traffic rules for implicit coordination, depending on, e.g. the geometry of a conflict which automatically result in coordinated manoeuvres.

The simplest implicit coordination could have been a binary choice: using priority rules, which gives one vehicle the freedom to manoeuvre in any way, as is common in road traffic. Using a priority rule, however, does take away the fail-safe aspect of decentralised separation assurance, which was responsible for its robustness by having two actors solving each conflict.

Another straightforward way to ensure implicitly coordinated manoeuvres, which maintains the fail-safe aspect, is used by MVP. In a conflict between two aircrafts, when the relative speed is changed in the direction away from the position of the other aircraft to move it outside the velocity obstacle, implicit coordination is also ensured. The resulting change in the ownship velocity vector corresponds to the shortest way out of the velocity obstacle. The direction of this avoidance vector is always away from the intruder's position during the closest point of approach. For the intruder, using the same logic on the conflict situation from its perspective automatically ensures an avoidance vector point away from the other avoidance manoeuvre as it always enlarges the distance and points away from the other's position at CPA. This results coordinated manoeuvring by both aircrafts without the need for any negotiation or coordination. Each vehicle assumes the other one will not manoeuvre and for fail-safe purposes, as the intruder might use a different dimension (vertical instead of horizontal), in which case the manoeuvre still ensures separation for one-on-one conflicts.

\section{Multiple Aircraft Conflicts and Summing Avoidance Vectors}

By definition, a conflict involves only two aircrafts, as it relies on the distance between two aircrafts predicted to be less than the minimally required separation assurance. When an aircraft is simultaneously in conflict with two aircrafts, it means that the separation will be lost within the lookahead time twice but with different intruders. When both conflict intervals are within the lookahead time, this classifies as a multi-aircraft conflict. Note that the other aircraft might experience this same conflict only as a single aircraft conflict. A multi-aircraft conflict depends on the perspective. Still, it could also be part of a whole cluster or a string of conflicts. When geometrical rules are used and the resulting resolution manoeuvres change this geometry, complex chain reactions can evolve, for which defining specific rules can be very challenging as the global, macroscopic behaviour is emergent behaviour.

A feature that distinguishes MVP from the other resolution algorithms in tables in [16••] is that it does not look for a conflict-free space in the solution space diagram, but treats each conflict independently and in case of multiple conflicts, sums the avoidance vectors. When avoidance vectors are pointing in opposing directions, or have an angle larger than $90^{\circ}$, the resulting initial manoeuvre will not completely solve the most urgent conflict, but relies on the implicit coordination to have the conflict partially solved by the intruder.

As the SSD shows the conflict-free speeds for one vehicle based on its surrounding traffic, making an algorithm that can resolve all conflicts using this two-dimensional map for finding speeds can be done in many ways. This seems a more advanced way to solve conflicts.

The MVP method relies on the macroscopic behaviour that it borrows from the "charged repelling particles" analogy and on its iterative approach to obtaining the final resolution manoeuvre. Intuitively, methods that opt for a joint solution 
would be preferable to this unpredictable strategy of the MVP algorithm, but recent studies have shown that, for multiaircraft conflicts, taking such a joint solution strategy comes at the cost of implicit coordination [17••].

\section{Comparing Macroscopic Performance of Different Geometric Resolution Algorithms}

To compare MVP solution with other conflict resolution, manoeuvres can also be visualised in a state-space diagram, which shows the velocity obstacles of the surrounding vehicles as was explained before [17••].

While the modified voltage potential method sums avoidance vectors, each of these avoidance vectors can be visualised as the shortest way out of the velocity obstacle. Because of the summing, the final MVP conflict resolution speed vector does not necessarily point to a free, open space in the SSD in case of multi-aircraft conflicts.

Therefore, in several studies, more advanced options to account for multi-aircraft conflicts were explored. Balasooriyan [17••] among others have generated multiple options of these manoeuvres, as shown in Fig. 5, which can also be regarded as a span of the many proposed conflict resolution algorithms in literature, as many fall in between [21].

The different algorithms were implemented and randomised high density scenarios were tested using 3000 fast-time simulation in the open source, open data air traffic simulator BlueSky $[22,23]$. The results of the fast-time simulations by Balasooriyan [17••] are shown in Fig. 6 .

Figure 6 shows the results of a recent fast-time simulation study [17••], which illustrates that even with the application of different coordination strategies to joint resolution methods, they still do not manage to outperform the combination of pairwise summing strategy of MVP and implicit coordination.
Later research also indicated that the moment when the recovery manoeuvre is initiated has an effect, meaning the DEP for MVP can be even further improved [24]

What is causing this macroscopic effectiveness of MVP even though at the microscopic scale, from the perspective of one aircraft, the more advanced SSD-based solutions appear to be better at solving conflicts?

\section{Hypothesised Cause for Macroscopic MVP Performance}

One possible explanation, which is hypothesised to be a unique feature, can be illustrated by the using synthetic conflict geometries. Even though these are not realistic traffic situations, they can serve as a metaphor for bottleneck situations in locally extremely high traffic densities. One very common synthetic multi-aircraft conflict geometry is the circular conflict, also called the superconflict. Several aircrafts are placed at an equal distance from the centre, evenly spread over a circle or one or more parts of the circle. This geometry is easily solved by most algorithms as, for example, shown in $[21,25]$.

A more challenging conflict geometry is a geometry named "the wall", in this horizontal conflict, a line of aircraft fly in the same direction next to each other separated only by the minimum separation $\mathrm{n}$ distance, forming a seemingly impenetrable wall of aircraft for one aircraft flying towards them in the opposite direction [8].

In this case, using a method, which looks for an open space in the solution space diagram for the opposing aircraft, could easily result in flying around the wall, or, in case of a very long wall, even turning more than $90^{\circ}$ to be able to avoid this wall of aircraft. Note that using priority rules could also result in the wall remaining intact.
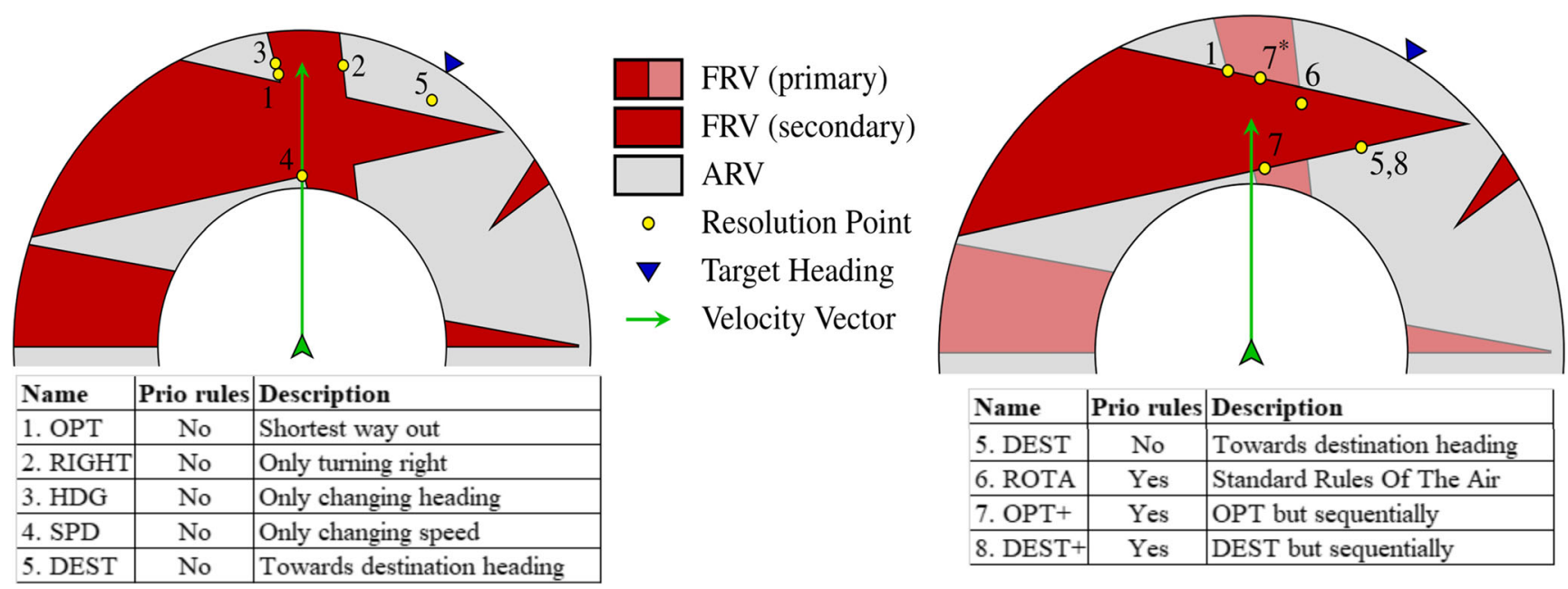

Fig. 5 Different resolution algorithms which have been compared to MVP visualised in an SSD with forbidden reachable velocities (FRV) and allowed reachable velocities (ARV) 
Fig. 6 Comparison of different resolution algorithms along different metrics shows that MVP outperforms the other algorithms in high traffic densities
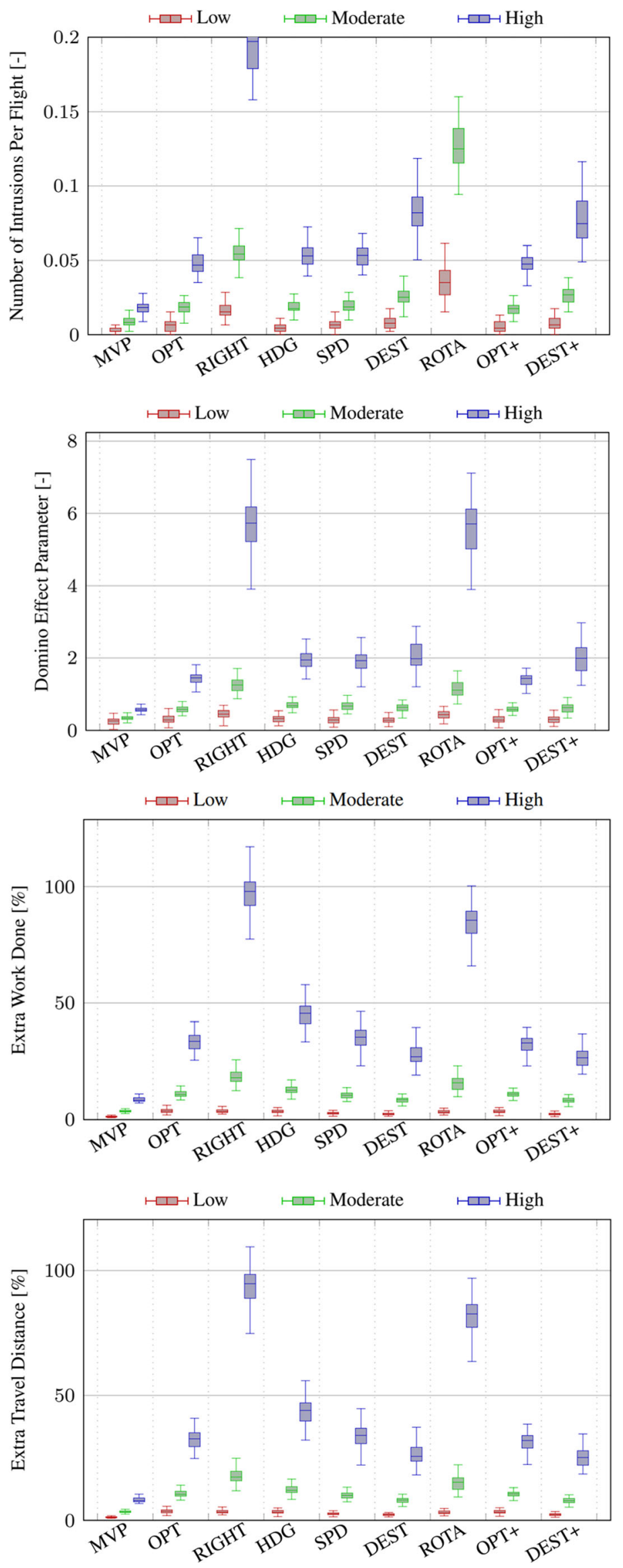
Fig. 7 MVP solution to "wall" scenario
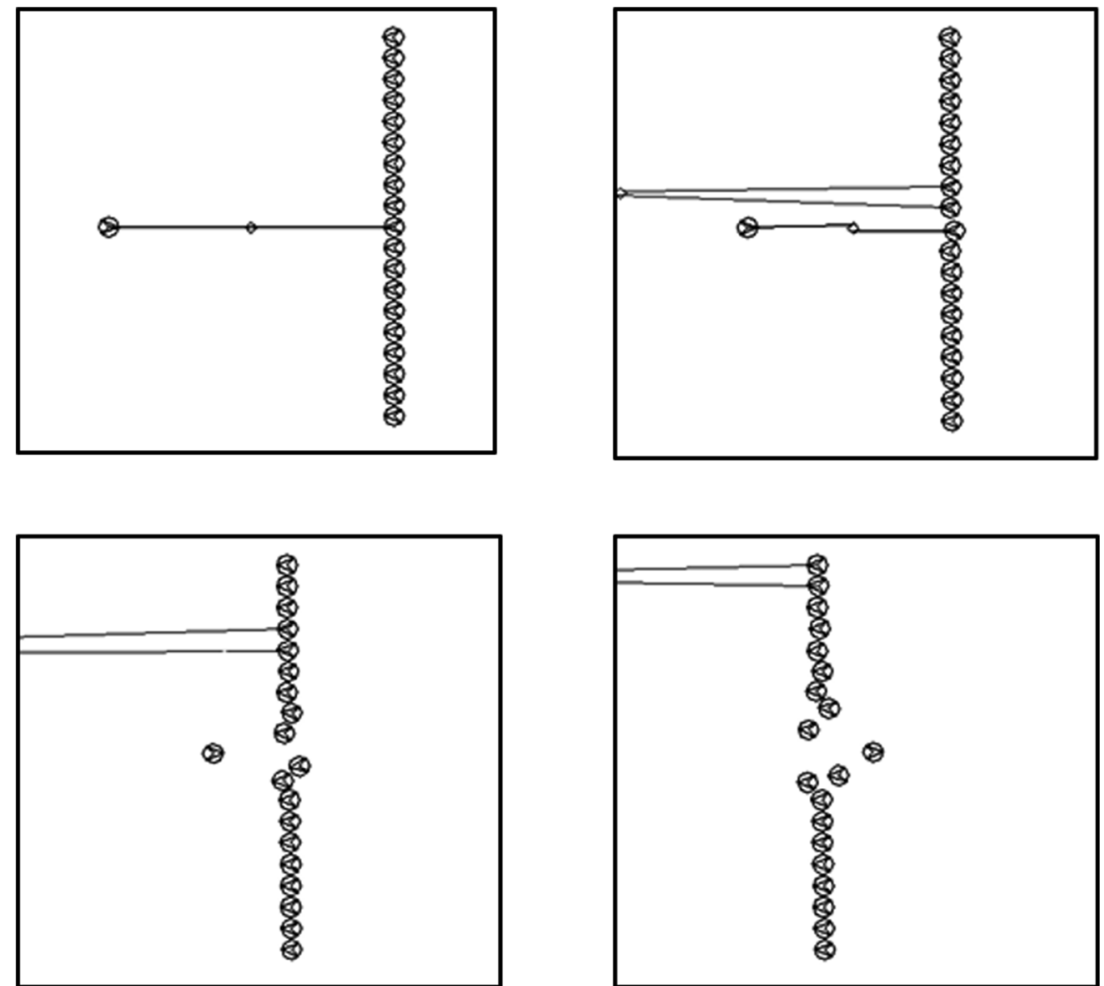

As is visible in Fig. 7, the MVP algorithms solve this synthetic conflict situation by creating a wave which ripples through the wall. This wave creates a hole in the wall for the opposing aircraft to go through. Although for some aircraft this means a sequence of two or three conflict alerts, the magnitude of the manoeuvre(s) is relatively small, just as the deviation from the route. In other words, using this tactical MVP resolution algorithm locally in a decentralised manner results in an efficient and effective global solution for the wall scenario. The feature of summing the avoidance vectors is responsible for this effective behaviour which is hypothesise to be responsible for the effectiveness of MVP in extreme traffic densities, as it distinguishes it from the other implemented options.

The MVP algorithm is particularly well adapted to urban air traffic scenarios, because of the expected extreme traffic densities and high concentration of traffic in that situation [26, 27] [28].

\section{Conclusion}

The research performed so far points in the direction of an urban airspace concept:

- Distributed separation assurance, i.e. detect and avoid, control due to the high number of vehicles and unpredictability of the flights
- Implicit coordination is a means to avoid bandwidth problems or deadlocks which can result in explicit coordination

- Co-operative conflict resolution is more robust than priority rules

- Use common air traffic rules, MVP has so far shown to be most promising robust conflict resolution algorithm with respect to solving bottlenecks, preventing losses of separation and maintaining efficient flights

While in manned flight a paradigm shift towards more trajectory-based operations is foreseen, the U-space or UTM require another paradigm change towards distributed control and central capacity management. Some research on capacity management based on traffic complexity [29-31], urban airspace structures $[26,32 \cdot]$ and geovectoring [33•] has been performed. But certainly more research in these areas is needed to fully understand the potential of, e.g. alignment, heading layers and segmentation and to find ways to ensure safety, capacity and efficiency in future phases of the implementation of urban airspace [34].

Acronyms and Symbols 4D-TBO, 4D trajectory-based operations; $\mathrm{ARV}$, allowed reachable velocities; CD\&R, conflict detection and resolution; CPA, closest point of approach; FRV, forbidden reachable velocities; MVP, modified voltage potential; PAV, personal air vehicles; TBO, trajectory-based operations; UAV, unmanned aerial vehicle; UTM, UAV traffic management (=U-space in Europe) 


\section{Declarations}

Conflict of Interest The authors declare no competing interests.

Human and Animal Rights and Informed Consent This article does not contain any studies with human or animal subjects performed by any of the authors.

Open Access This article is licensed under a Creative Commons Attribution 4.0 International License, which permits use, sharing, adaptation, distribution and reproduction in any medium or format, as long as you give appropriate credit to the original author(s) and the source, provide a link to the Creative Commons licence, and indicate if changes were made. The images or other third party material in this article are included in the article's Creative Commons licence, unless indicated otherwise in a credit line to the material. If material is not included in the article's Creative Commons licence and your intended use is not permitted by statutory regulation or exceeds the permitted use, you will need to obtain permission directly from the copyright holder. To view a copy of this licence, visit http://creativecommons.org/licenses/by/4.0/.

\section{References}

Papers of particular interest, published recently, have been highlighted as:

- Of importance

•. Of major importance

1. Narkus-Kramer, Marc P. (2017), Future demand and benefits for small unmanned aerial systems (UAS) package delivery. 17th AIAA aviation technology, integration, and operations conference, 1-7, URL arc.aiaa.org/doi/10.2514/6.2017-4103

2. ICAO, July. "Air traffic services: ANNEX 11 to the convention of international civil aviation", Chapter 2 Section 2.6, (2001)

3. RTCA. Task Force3, "Free flight implementation, final report of RTCA Task Force 3”. Washington, DC: RTCA; 1996.

4. Wing D, Krishnamurthy K, Barhydt R, Barmore B. Pilot interactions in an over-constrained conflict scenario as studied in a piloted simulation of autonomous aircraft operations, 5th USA/Europe ATM R\&D seminar. Hungary: Budapest; 2003.

5. Blom HA, Krystul J, Bakker GJ, Klompstra MB, Obbink BK. Free flight collision risk estimation by sequential MC simulation. Stochastic hybrid systems. 2007:249-81.

6. Blom HA, Bakker GJ. Safety evaluation of advanced selfseparation under very high en route traffic demand. Journal of Aerospace Information Systems. 2015;12(6):413-27.

7. Hoekstra JM, van Gent RN, Ruigrok RC. Designing for safety: the 'free flight' air traffic management concept. Reliab Eng Syst Saf. 2002;75(2):215-32.

8. Hoekstra JM, Ruigrok RC, Van Gent RN. Free flight in a crowded airspace? in Proceedings of the 3rd USA/Europe air traffic management R\&D seminar 2001

9. SESAR, The roadmap for delivering high performing aviation for Europe, European ATM master plan. Executive view. Edition 2015.

10. SESAR, SESAR concept of operations step 1. D65-011. Project B4.2.
11. Erzberger H, Paielli RA, Isaacson DR, Eshow MM. Conflict detection and resolution in the presence of prediction error. In1st USA/ Europe air traffic management R\&D seminar, Saclay, France 1997 Jun 17 (pp. 17-20)

12. Fiorini $\mathrm{P}$, Shiller Z. Motion planning in dynamic environments using velocity obstacles. The International Journal of Robotics Research. 1998 Jul;17(7):760-72.

13. Ellerbroek, J. "Airborne conflict resolution in three dimensions." Doctoral thesis TU Delft (2013)

14. Kuchar J, Yang L. A review of conflict detection and resolution modeling methods. IEEE Trans Intell Transp Syst. 2000;1:179-89.

15. Jenie YI, Van Kampen EJ, Ellerbroek J, Hoekstra JM. Taxonomy of conflict detection and resolution approaches for unmanned aerial vehicle in an integrated airspace. IEEE Trans Intell Transp Syst. 2016 Jul 22;18(3):558-67.

16.• Ribeiro M, Ellerbroek J, Hoekstra J. Review of conflict resolution methods for manned and unmanned aviation. Aerospace. 2020;7:79 This paper contains an extensive inventarisation of more than 100 conflict resolution methods based on a taxonomy which is explained in the paper.

17.• Balasooriyan S. Multi-aircraft conflict resolution using velocity obstacles, MSc Thesis, TU Delft, 2017. This paper has developed variations of modified voltage potential (MVP) and similar state-based algorithms. The original MVP with the summing of avoidance vectors shows better performance than tailored optimisation or advanced variations.

18. Krozel J, Peters M, Bilimoria KD, Lee C, Mitchell JS. System performance characteristics of centralized and decentralized air traffic separation strategies. Air Traffic Control Quarterly. 2001 Oct;9(4):311-32.

19. Eby M. A self-organizational approach for resolving air traffic conflicts. Linc Lab J. 1994;7:2

20. Hoekstra, J. Designing for safety: the free flight air traffic management concept. Ph.D. Thesis, Delft University of Technology, Delft, The Netherlands, 2001

21. Durand N. Constant speed optimal reciprocal collision avoidance. Transportation research part C: emerging technologies. 2018 Nov 1;96:366-79.

22. Hoekstra JM, Ellerbroek J. Bluesky ATC simulator project: an open data and open source approach. In Proceedings of the 7th international conference on research in air transportation 2016 Jun (Vol. 131, p. 132). USA/Europe: FAA/Eurocontrol.

23. BlueSky GitHub repository https://github.com/TUDelft-CNSATM/bluesky

24. Schaberg W. A decentralized recovery method for air traffic conflicts. TU Delft: MSc Thesis; 2020.

25. Delahaye D, Peyronne C, Mongeau M, Puechmorel S. Aircraft conflict resolution by genetic algorithm and B-spline approximation

26. Sunil E, Hoekstra J, Ellerbroek J, Bussink F, Nieuwenhuisen D, Vidosavljevic A, Kern S. Metropolis: relating airspace structure and capacity for extreme traffic densities

27. Doole M, Ellerbroek J, Hoekstra J. Estimation of traffic density from drone-based delivery in very low level urban airspace. J Air Transp Manag. 2020 Sep 1;88:101862.

28. SESAR. Joint Undertaking. SESAR, Brussels: European drones outlook study. Unlocking the value for Europe; 2016.

29. Laudeman IV, Shelden SG, Branstrom R, Brasil CL. Dynamic density: an air traffic management metric.

30. Flynn GM, Leleu C, Hilburn B. A complexity study of the Maastricht Upper Airspace Centre. EEC Report. 2006;403. 
31. Sunil E, Ellerbroek J, Hoekstra JM. CAMDA: Capacity assessment method for decentralized air traffic control. International conference for research of air transport (ICRAT 2018), Barcelona 2018

32. Hoekstra JM, Maas J, Tra M, Sunil E. How do layered airspace design parameters affect airspace capacity and safety? In Proceedings of the 7th international conference on research in air transportation 2016 Jun. The paper explains the principle of conflict prevention by segmentation and relative speed reduction based on a mathematical analysis.

33. Hoekstra JM, Ellerbroek J, Sunil E, Maas J. Geovectoring: reducing traffic complexity to increase the capacity of UAV airspace. International conference for research in air transportation, Barcelona, Spain 2018. This paper explains how findings from [34] can be translated into technology requirements for an urban UAV ATM system, using a new principle called geovectoring.

34. Alarcón V, García M, Alarcón F, Viguria A, Martínez Á, Janisch D, et al. Procedures for the integration of drones into the airspace based on U-space services. Aerospace. 2020 Sep;7(9):128.
35.• Ribeiro M, Ellerbroek J, Hoekstra J. Analysis of conflict resolution methods for manned and unmanned aviation using fast-time simulations. 9th SESAR innovation days 2nd-5th December 2019. 2019. This paper contains a comparison of several conflict resolution/avoidance algorithms using fast-time simulations. Velocity obstacles-based methods perform better than other methods, of these, the modified voltage potential (MVP) appears as the most promising.

36. Doole M, Ellerbroek J, Hoekstra J, Mennella A, Onate M. Drone information service requirements for U-space. 8th SESAR innovation days, 2018. 2018.

Publisher's Note Springer Nature remains neutral with regard to jurisdictional claims in published maps and institutional affiliations. 